\title{
Roles of Two Sox9 Genes during Gonadal Development in Japanese Flounder: Sex Differentiation, Spermatogenesis and Gonadal Function Maintenance
}

\author{
Xiaojing Li, Haiyang Yu, Yujue Wang, Xiaobing Liu, Yuezhong Liu, Jiangbo Qu and Xubo Wang * \\ Ministry of Education Key Laboratory of Marine Genetics and Breeding, College of Marine Life Sciences, \\ Ocean University of China, Qingdao 266003, China; lixiaojing920825@163.com (X.L.); \\ yuhaiyangyx@163.com (H.Y.); wangyujue1236@126.com (Y.W.); liuxiaobing132@163.com (X.L.); \\ yuezhong_liu@163.com (Y.L.); jbq0315@163.com (J.Q.) \\ * Correspondence: wangxubo@ouc.edu.cn; Tel.: +86-532-8203-1986
}

Received: 7 December 2017; Accepted: 22 January 2018; Published: 8 February 2018

\begin{abstract}
The transcription factor sox 9 has been implicated in cartilage formation and testis determination in mammals. Here, two duplicates of sox 9 were found in Japanese flounder (Paralichthys olivaceus) named Posox9a and Posox9b, respectively. Phylogenetic and gene structure analyses revealed that Posox9a and Posox $9 b$ were homologous to that of teleosts and tetrapods. Quantitative real-time polymerase chain reaction (qRT-PCR) showed that both Posox $9 a$ and Posox $9 b$ expressed higher in testis than in ovary of adult tissues. The in situ hybridization (ISH) analysis of gonads showed that Posox $9 a$ and Posox $9 b$ mRNA were both detected in oocytes, Sertoli cells and spermatocytes. During sex differentiation, the expression of Posox9a exhibited obvious sexual dimorphic expression from 60 days after hatch (dah) with higher expression in male preferred individuals than female preferred individuals and increased gradually from 30 to 100 dah. A similar pattern was detected in Posox $9 b$ expression. After injection of androgen ( $17 \alpha$-methyltestosterone) of different concentrations, the expression level of Posox $9 \mathrm{~b}$ increased significantly, whereas Posox9a did not change obviously. These results indicated that the two sox 9 genes of Japanese flounder had converse functions in sex differentiation, whereas their differences in $17 \alpha$-methyltestosterone administration were obvious and worthwhile for exploring evolutionary and adaptive significance. This study provided a foundation for further exploration of the roles of Posox 9 genes during the sex determination and differentiation, spermatogenesis and gonadal function maintenance of Japanese flounder.
\end{abstract}

Keywords: Japanese flounder; Posox9a and Posox9b; dimorphic expression; sex differentiation; $17 \alpha$-Methyltestosterone administration; gonadal development

\section{Introduction}

Research on the Sox gene family began with the seminal discovery of the mammalian testis-determining factor-SRY [1,2]. The identification and homology-based analysis of the high mobility group (HMG), DNA-binding domain of SRY led to the discovery of the Sry-related HMG box (SOX) transcription factor family [3]. In general, proteins containing an HMG domain with $60 \%$ or higher amino acid similarity to the HMG domain of SRY are referred as Sox proteins [4]. Sox proteins that share an HMG domain with more than $80 \%$ sequence identity are divided into different HMG groups termed A to $\mathrm{H}$ [5]. 
Sox9 gene, a member of SoxE subfamily, is a transcription factor required for cartilage formation and testis determination in mammals [6,7]. SRY initiates a cascade of gene networks through the direct regulation of Sox 9 expression and promotes supporting cell differentiation, Leydig cell specification, vasculature formation and testis cord development [8]. Mutations in the human SOX9 gene cause skeletal defects and male-to-female sex reversal, indicating its essential roles in chondrogenesis and testis development. In mice and chickens, the expression of Sox9 is down-regulated in the differentiating gonad just before ovary formation, but its expression in Sertoli cells of the developing testis persists throughout adulthood [9-11]. Two sox9 genes have been found in several teleost species, such as zebrafish (Danio rerio), fugu (Takifugu rubripes), stickleback (Gasterosteus aculeatus), rice field eel (Monopterus albus) and rainbow trout (Oncorhynchus mykiss). Zebrafish sox9a transcripts have been detected in Sertoli cells of the testis, while sox $9 b$ has been detected in ovaries [12]. Fugu sox $9 a$ expresses at higher levels in the testis than in the ovary, whereas its sox $9 b$ is only detected in the ovary [13]. In rice field eel, both sox9a1 and sox9a2 express in the testis, ovary, as well as in the ovotestis of intersex individuals. In sablefish (Anoplopoma fimbria), sox 9 mRNA prominently expresses in the testis, which indicates that sox 9 is related to the formation and differentiation of the testis [14]. This male-favored expression profile at the stage of testis formation is also observed in freshwater and marine turtles [15], denoting that the functional importance of sox 9 for male differentiation is conserved among tetrapods. Furthermore, with the ablation of Sox9, mice exhibit defects in the specification of oligodendrocytes and astrocytes, suggesting important roles of $\operatorname{sox} 9$ in the development of central nervous system [16]. In Xenopus, the depletion of SOX9 protein in developing embryos causes a dramatic loss of neural crest progenitors and an expansion of the neural plate, indicating that SOX9 is required for cranial neural crest development [17]. All of these findings implied that sox 9 is a multifunctional gene, especially in the differentiation and maintenance of the testis and the development of the central nervous system.

Because of the whole genome duplication event, two sox9 duplicates (named Posox9a and Posox9b) are found in Japanese flounder (Paralichthys olivaceus), which is one of the most important species in aquaculture with a stable XX/XY sex determination system [18]. In this study, we focus mainly on the molecular and genetic features of the two sox9 genes of Japanese flounder from analyses of larvae and adults in sex development, as well as the effects of androgen (17 $\alpha$-methyltestosterone) administration on their expression. The results will improve the current understanding of the expression patterns, biological functions and evolutionary significance of Japanese flounder sox 9 genes in sex differentiation, gonadal development and maintenance.

\section{Results}

\subsection{Molecular Characterization of Posox9a and Posox9b}

\subsubsection{Cloning and Sequence Analysis of Posox9a and Posox9b}

Sequences of Posox $9 a$ and Posox $9 b$ were obtained from the genome and transcriptome of Japanese flounder by the local alignment search tool (BLAST+2.6.0). Besides, the full-length coding region of the two sequences was confirmed by amplifying, cloning, and sequencing and they were significantly similar to other sox $9 a$ and sox $9 b$ genes by BLAST search at National Center of Biotechnology Information (NCBI), respectively. Comparison of genomic and cDNA sequences showed that both Posox $9 a$ and Posox $9 b$ contained three exons and two introns. The predicted amino acid sequences of PoSox9a and PoSox $9 \mathrm{~b}$ were 497 and 478 residues respectively. Based on bioinformatics analysis, highly conserved HMG-box domains of the Sox superfamily was found in PoSox9a and PoSox9b sequences from residue 103-169 and 102-169, respectively (Figure 1). 


\subsubsection{Homology and Phylogenetic Analysis}

To evaluate the evolutionary relationship between the predicted PoSox9a/b and another vertebrate group E Sox, a phylogenetic tree was generated using a maximum likelihood algorithm by MEGA6.0 with a Whelan and Goldman (WAG) model based on the amino acid sequences analyzed by MUSCLE3.8.31 (Figure 2). HMG domains showed a higher percentage of identity value among different species than other domains (Figure 1A,B). Moreover, since the HMG domain in the PoSox9a and PoSox9b protein were highly conserved, the genetic and evolutionary diversity in Sox 9 among species was caused by the variety of the peptide sequences other than the HMG domain.

\subsubsection{Genomic Structure of Posox9 Genes}

The genomic structures of sox 9 genes in many vertebrates were determined based on their published whole-genome sequences. The Posox 9 genes structures were compared with other vertebrates. As the untranslated region (UTR) sequences of various species were incomplete, we only focused on the open reading frame (ORF) sequences. Analysis of the genomic structure showed that both Posox $9 a$ and Posox9b ORF contained three exons and two introns (Figure 3). Compared with the sox 9 of other species, the genomic structures of the two duplicates of Japanese flounder sox 9 were conserved. All exon-intron boundaries were consistent with the 5'-GT and 3'-AG splicing rule. These results suggested that the two duplicates of Japanese flounder sox 9 genes exhibited a conserved number of exons between flounder species and other tetrapods despite their different gene size.

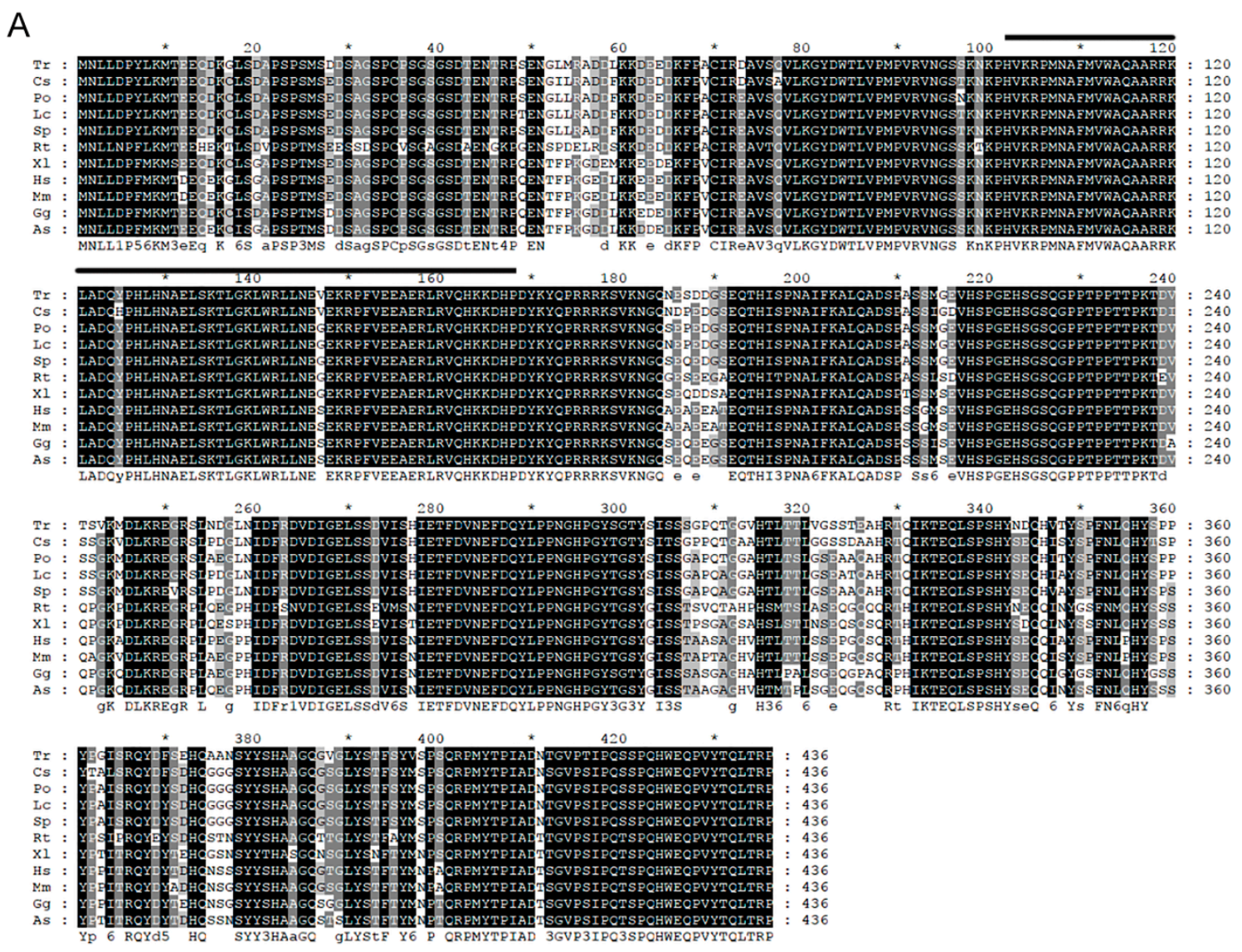

Figure 1. Cont. 
B
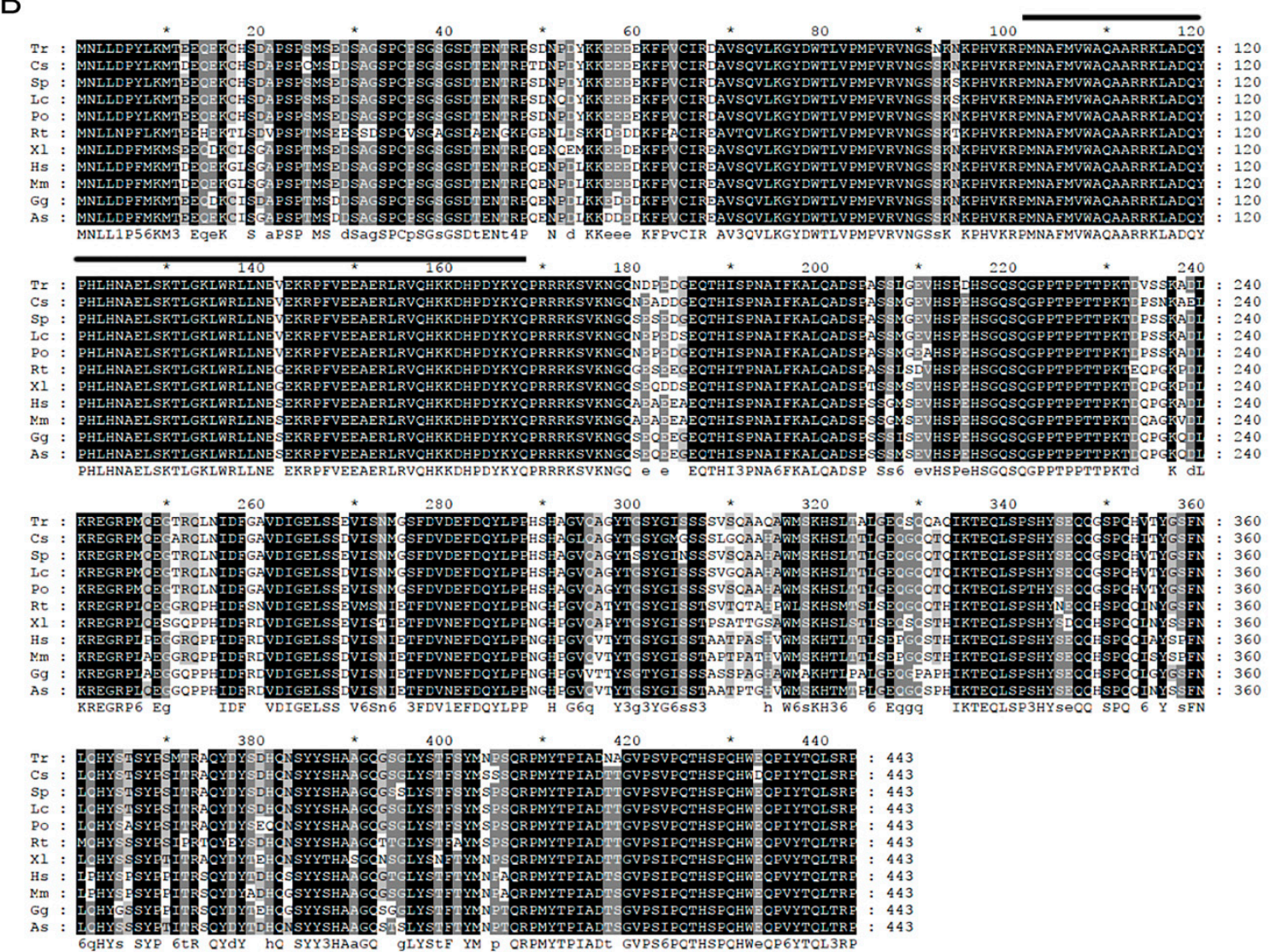

Figure 1. Multiple alignment of Sox9 amino acid sequences in different species. (A) PoSox9a; (B) PoSox9b. The black shadow region indicates positions where all sequences selected share the same amino acid residue. Identical amino acids are in dark grey background. The conserved regions are indicated with black lines. GenBank accession numbers of these sequences are as follows: Takifugu rubripes Sox9a (Tr, AAQ18508.1); Cynoglossus semilaevis Sox9a (Cs, XP_008313399.1); Stegastes partitus Sox9a (Sp, XP_008295125.1); Lates calcarifer Sox9a (Lc, XP_018541051.1); Paralichthys olivaceus Sox9a (Po, unpublished); Rhincodon typus Sox9a (Rt, XP_020382797.1); Xenopus laevis Sox9 (X1, NP_001087942.1); Homo sapiens SOX9 (Hs, NP_000337.1); Mus musculus Sox9 (Mm, NP_035578.3); Gallus gallus Sox9 (Gg, NP_989612.1); Alligator sinensis Sox9 (As, XP_006029531.1); Takifugu rubripes Sox9b (Tr, AAL32172.1); Cynoglossus semilaevis Sox9b (Cs, XP_016895678.1); Stegastes partitus Sox9b (XP_008301579.1); Lates calcarifer Sox9b (Lc, XP_018541051.1); Paralichthys olivaceus Sox9b (Po, ACO40490.1); Rhincodon typus Sox9b (Rt, XP_020382797.1).

\subsection{Relative Expression of Posox9a and Posox $9 b$ in Different Tissues}

Tissue-specific expression of Posox $9 a$ and Posox $9 b$ in adult Japanese flounder was analyzed by qRT-PCR. Posox9a gene expressed at higher levels in the muscle, testis and gill, and at lower levels in the liver, kidney and brain; the transcripts were nearly undetectable in the heart, spleen, intestine and ovary (Figure 4A). Notably, the Posox9a expression level in the testis was significantly higher than in the ovary. The Posox $9 b$ gene expressed at higher levels in the brain and gill, and at lower levels in the intestine and testis; the transcripts were nearly undetectable in the heart, liver, spleen, kidney, muscle, intestine and ovary (Figure 4B). Notably, the Posox $9 b$ gene also expressed at higher levels in the testis than ovary, which was similar to the expression of Posox9a in adult gonads. 


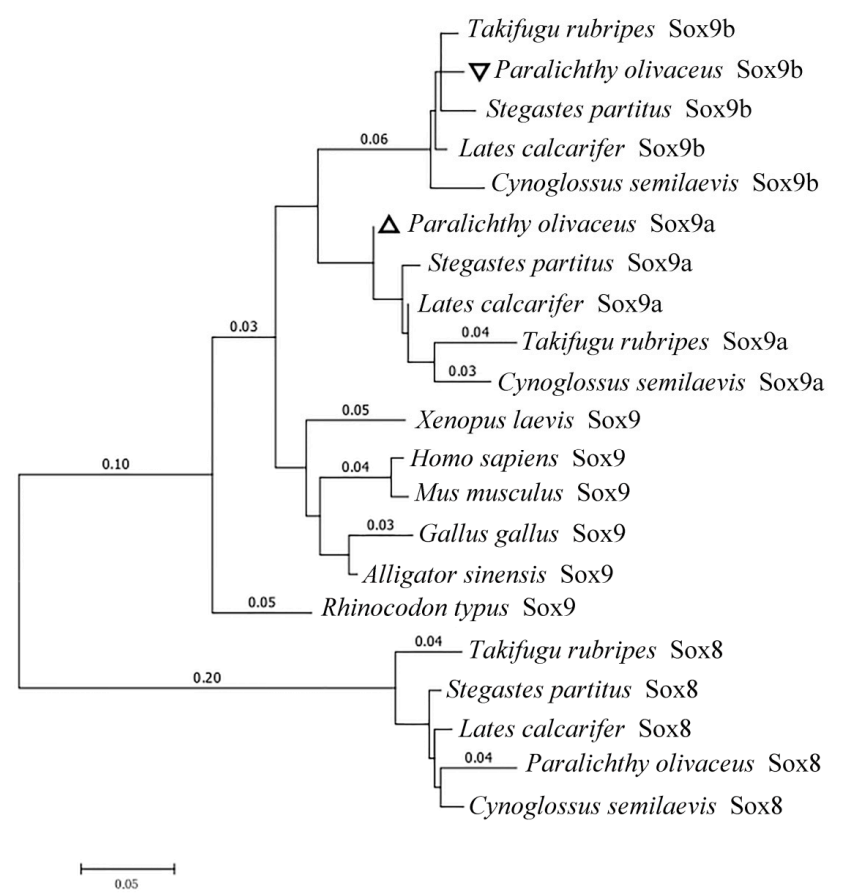

Figure 2. Phylogenetic tree of PoSox9a and PoSox9b in comparison with Sox9 and Sox 8 proteins in other representative vertebrates using predicted amino acid sequences. The two triangular symbols in the picture indicate the two Sox9 in Paralichthy olivaceus. The phylogenic tree was generated by MEGA6.0 with the WAG model. The scale bar is 0.05 . The GenBank accession numbers are as follows: Homo sapiens Sox9, NP_000337.1; Mus musculus Sox9, NP_035578.3; Gallus gallus Sox9, NP_989612.1; Alligator sinensis Sox9, XP_006029531.1; Xenopus laevis Sox9, NP_001087942.1; Rhincodon typus Sox9, XP_020382797.1; Lates calarifer Sox9a, XP_018541051.1; Stegastes partitus Sox9a, XP_0082905125.1; Paralichthys olivaceus Sox9a, unpublished; Cynoglossus semilaevis Sox9a, XP_008313399.1; Takifugu rubripes Sox9a, AAQ18508.1; Cynoglossus semilaevis Sox9b, XP_016895678.1; Stegastes partitus Sox9b, XP_008301579.1; Lates calarifer Sox9b, XP_018536323.1; Takifugu rubripes Sox9b, AAL32172.1; Paralichthys olivaceus Sox9b, ACO40490.1; Lates calarifer Sox8, AKI32583.1; Stegastes partitus Sox8, XP_008301648.1; Cynoglossus semilaevis Sox8, XP_008328025.1; Takifugu rubripes Sox8, NP_001072112.1.
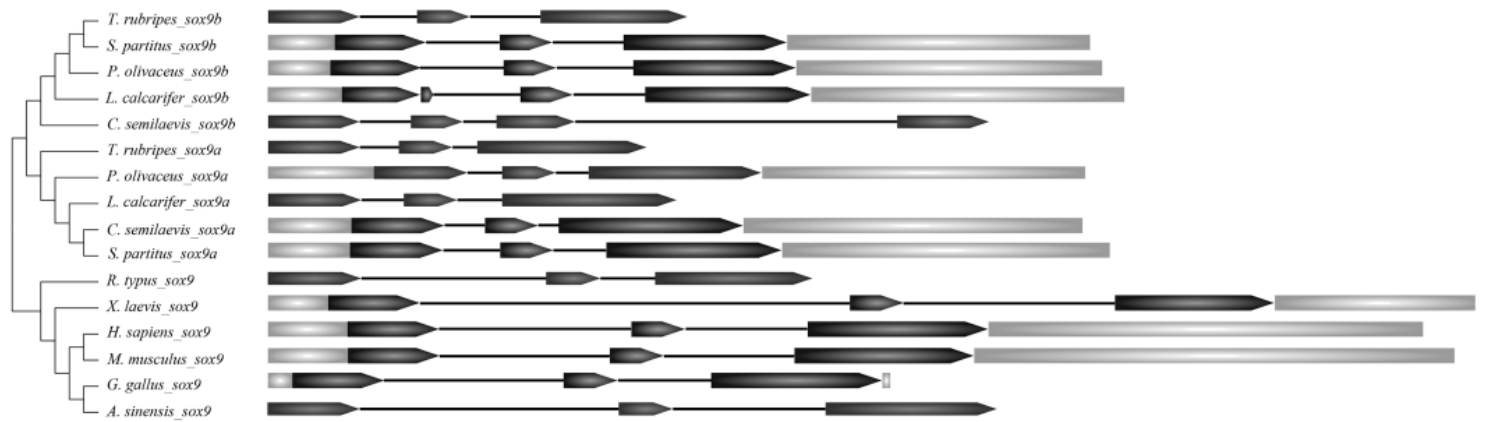

Legend:

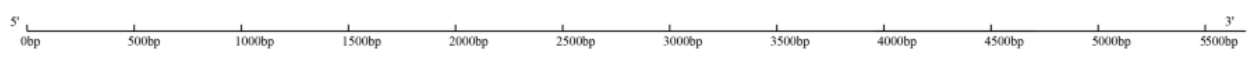

- CDS D UTR - Intron

Figure 3. Comparison of genomic organizations of sox9 between teleosts and tetrapods. Exons are shown in the black wedge, introns are shown in the straight line, and untranslated regions (UTRs) are shown in the gray box. GenBank accession numbers of these sequences are the same as those used in the phylogenetic analysis. 

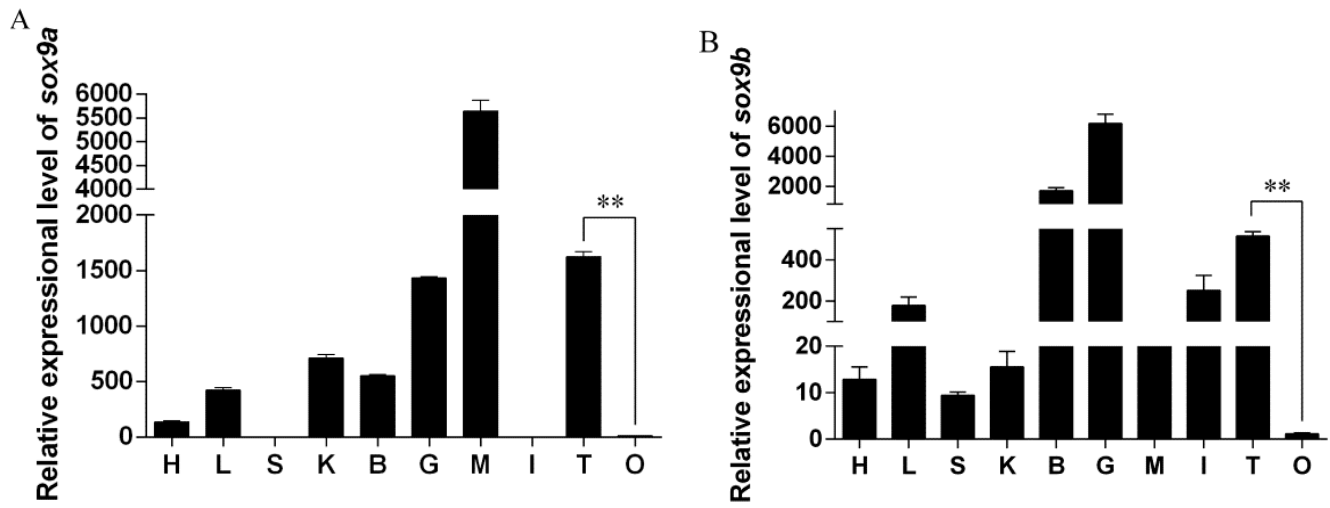

Figure 4. Expression of Posox $9 a$ and Posox $9 b$ mRNA in tissues quantified by qRT-PCR. (A) Posox9a; (B) Posox9b. Abbreviations: H: heart; L: liver; S: spleen; K: kidney; B: brain; G: gill; M: muscle; I: intestine; T: testis; O: ovary. Data are shown as mean \pm Standard Error of Mean (SEM) $(n=3)$.

** indicates statistical significance $(p<0.01)$.

The distribution of Posox $9 a$ and Posox9b mRNA in gonadal sections were detected by in situ hybridization (ISH) (Figure 5) using a Digoxin (DIG)-labeled, anti-sense RNA probe. In the adult testis, the cells were mainly comprised of Sertoli cells, spermatocytes and spermatids. ISH results showed that strong positive signals of Posox $9 a$ and Posox $9 b$ mRNA were both found in Sertoli cells and spermatocytes (Figure 5A-b,B-b). No signal was detected in spermatids. As for the adult ovary, the cells were mainly comprised of oogonia and oocytes. The signal of Posox9a and Posox $9 \mathrm{~b}$ mRNA were both detected in the cytoplasm of the oocytes (Figure 5A-e,B-e). No signal was found when detected with the sense probes (Figure 5A-c,f,B-c,f). Spatial expression results showed that Posox9a and Posox $9 b$ mRNA were almost detected in the same location in the gonads.

A
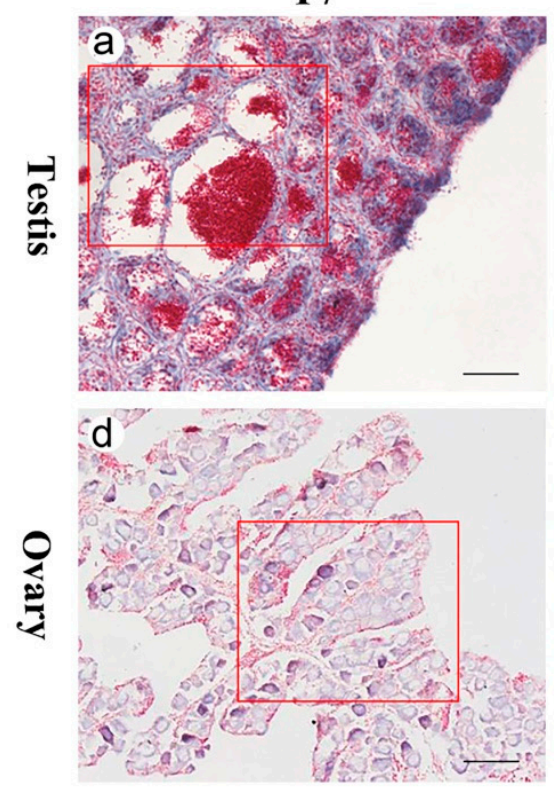

T7

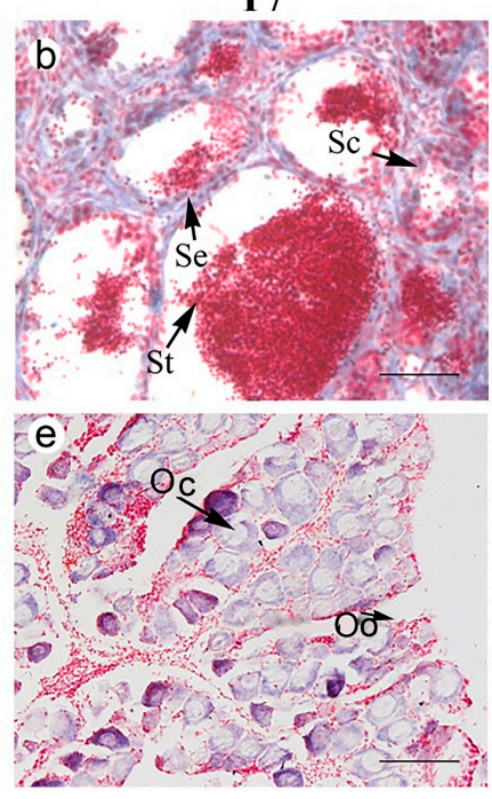

SP6
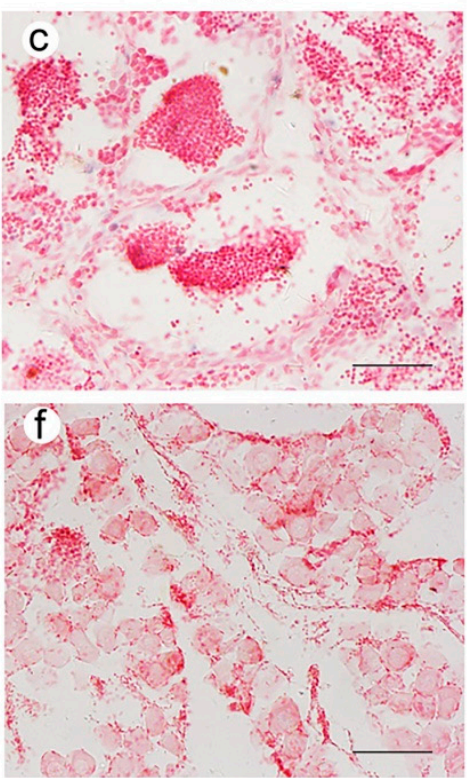

Figure 5. Cont. 
B
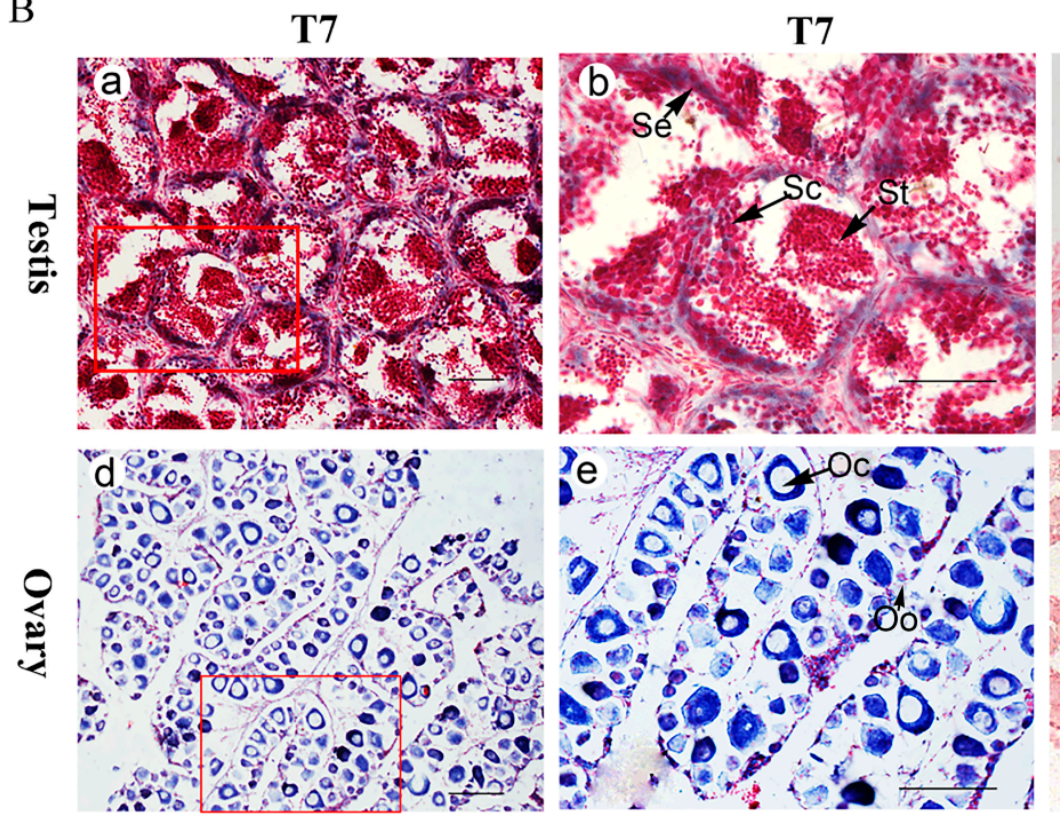

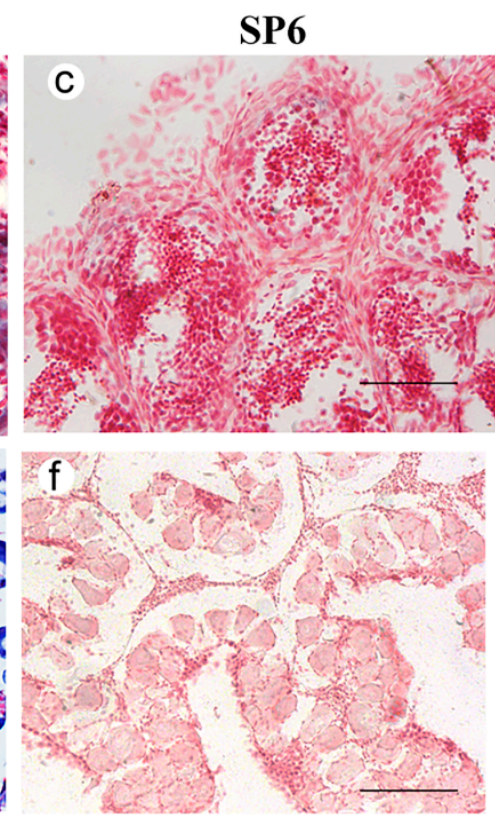

Figure 5. Expression of Posox $9 a$ and Posox $9 b$ mRNA in the gonads detected by ISH. (A) Posox9a; (B) Posox $9 b$. The positive signals were stained as purple or blue, whereas the negative control with sense probe hybridization was unstained. Abbreviations: Se, Sertoli cells; Sc, spermatocytes; St, spermatid; Oo, oogonia; Oc, oocytes. Scale bars $=100 \mu \mathrm{m}$.

\subsection{Sexually Dimorphic Expression of Posox9a and Posox9b Mrnas in Gonads}

Anti-Mullerian hormone ( $a m h)$, also known as Mullerian inhibiting substance (MIS), is responsible for the regression of Mullerian ducts, so it can lead to the masculinization of gonads. It has been reported that Japanese flounder amh is sexually dimorphic during sex gonadal differentiation [19]. For the lack of genetic gender marker in Japanese flounder, we referenced the expression of amh to distinguish the genetic male and female individuals [20]. The exact expression of $a m h$, Posox $9 a$ and Posox $9 b$ in gonads during the early development stage (30 to 100 dah) were analyzed by qRT-PCR. At 30 dah, when the gonad was sexually indistinguishable, amh RNA mainly expressed in five individuals (Figure 6A, Individual 2, 3, 6, 8 and 9), which were regarded as genetic males, but hardly expressed in the others (Figure 6A, Individual 1, 4, 5, 7 and 10), which were considered as genetic females. Neither Posox9a nor Posox $9 b$ showed obvious sexual dimorphic expression during this stage. After the initiation of sex differentiation ( $60 \mathrm{dah}$ ), higher levels of amh mRNA were detected in the five individuals (genetic males) than the others (genetic females) (Figure 6B). The expression levels of both Posox $9 a$ and Posox $9 b$ in these genetic males were significantly higher than in genetic females (Figure 6F,J). From 80 to 100 dah, the expression of $a m h$ mRNA increased remarkably in the gonad of genetic females, but was consistently lower in the genetic males (Figure 6C,D). Similarly, Posox9a and Posox $9 b$ at this stage expressed at higher levels than the stage of $30 \mathrm{dah}$, with an obvious sexual dimorphic pattern (Figure $6 \mathrm{G}, \mathrm{H}, \mathrm{K}, \mathrm{L}$ ). It is noteworthy that among all these stages, Posox $9 b$ expressed at higher levels than Posox $9 a$, especially at 30 dah. 

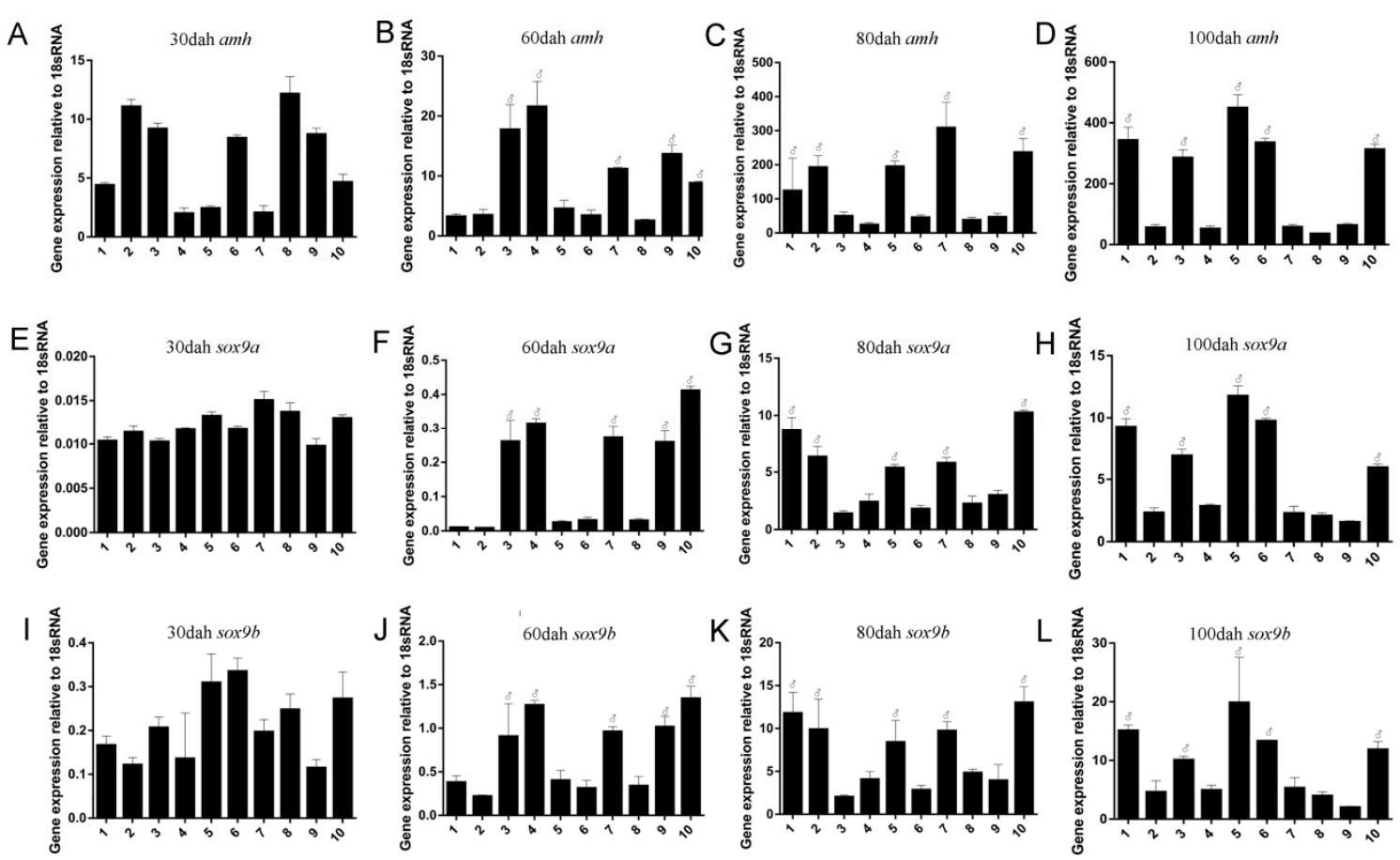

Figure 6. The expression of sox $9 a$ and sox $9 b$ genes during gonadal differentiation determined by qRT-PCR analysis. Genetic males and females were distinguished at 30, 60, 80 and 100 days after hatching (dah) according to the expression level of $a m h$ (A-D). Numbers 1 to 10 indicate ten individuals. The symbol $\sigma^{7}$ indicates genetic males, while the others are genetic females. (E-H) mRNA levels of sox $9 a$ in these corresponding genetic male and female gonads from 30 to 100 dah. (I-L) mRNA levels of sox $9 b$ in these corresponding genetic male and female gonads from 30 to $100 \mathrm{dah}$.

\subsection{Expression Profile of Posox9a and Posox9b after $17 \alpha$-methyltestosterone Injection}

After five days of injection of $17 \alpha$-methyltestosterone, the expression profiles of Posox $9 a$ and Posox $9 b$ were studied in adult Japanese flounder by qRT-PCR (Figure 7). The results showed that the expression level of Posox $9 b$ in the testis increased obviously by $17 \alpha$-methyltestosterone administration, especially when the $17 \alpha$-methyltestosterone concentration was $1.5 \mathrm{mg} / \mathrm{mL}$ (Figure 7B), whereas Posox $9 a$ exhibited no significant difference between the experiment groups and control group (Figure 7A).
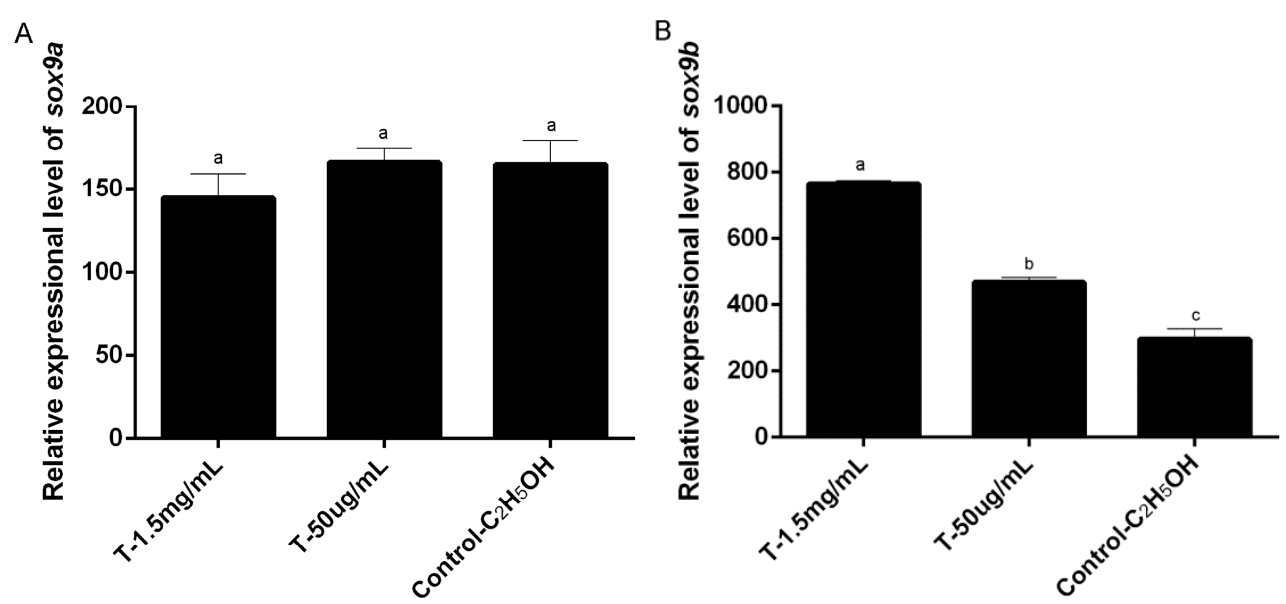

Figure 7. qRT-PCR analysis showing Posox $9 a$ and Posox $9 b$ mRNA levels in the testis. (A) Posox9a; (B) Posox $9 b$. Data of qRT-PCR were expressed as mean \pm SEM $(n=3)$. The same letter used between the groups indicates no significant difference $(p>0.05)$. 


\section{Discussion}

The Sox 9 gene is an important factor required for cartilage formation and testis determination in mammals [6,7]. In mice, sox9 is not required for testis cord differentiation after 14 days postcoitum, but is critical for the maintenance of adult testicular functions and fertility [21]. In recent years, more and more research has been performed on the sex determination genes of teleosts. Sox 9 is a candidate gene for the selection of sex determination gene. Japanese flounder, one of the most important species in aquaculture with a stable $\mathrm{XX} / \mathrm{XY}$ sex determination system, belongs to Pleuronectiformes, Paralichthyidae and Paralichthys. Considering its growth differences between male and female individuals and its economic benefits, constructing all-female stocks is of great value. Thus, the exploitation of Japanese flounder sex-related genes has become an important assignment for assisting breeding at the molecular level.

\subsection{Posox9a and Posox9b Are Highly Conversed in Vertebrates}

In this study, two duplicates of the sox 9 gene in Japanese flounder were found, supporting the understanding that sox 9 is duplicated in Japanese flounder, as has also been observed in other fish species, such as zebrafish, fugu and rice field eel due to the whole genome duplication event. Bioinformatic analysis revealed that the genomic structure of Posox $9 a$ and Posox $9 b$ were conserved. Evidence gathered from protein sequences and the conserved and characteristic domains demonstrated that Posox9a and Posox9b encoded Japanese flounder PoSox9a and PoSox9b respectively, and were most closely related to the corresponding homologues of uncovered Sox9 proteins. PoSox9a and PoSox9b, especially the sequences within the conserved HMG-box domain, shared high amino acid sequence identities with other species, implying that PoSox9a and PoSox9b might possess similar regulation and function mechanisms as their homologues.

\subsection{Both Posox9a and Posox9b Are More Abundant in Testis Than in Ovary}

The tissue-specific expression of Posox $9 a$ and Posox $9 b$ in adult Japanese flounder was investigated by qRT-PCR to determine whether the genes were uniformly expressed or restricted to specific tissues or genders. The Posox $9 a$ and Posox $9 b$ genes were widely expressed in the examined tissues and the expression profiles of the two duplicates were different: the Posox $9 a$ level was highest in the muscle and Posox $9 b$ in the gill, indicating they might play different roles in these tissues. Here, we focused on the roles of the two Posox 9 genes in the gonads. The qRT-PCR results showed that the expression levels of Posox $9 a$ and Posox $9 b$ were both higher in the testis than in the ovary. The same expression profile was also found in Anoplopoma fimbria [14], half-smooth tongue sole (Cynoglossus semilaevis) [22] and catfish sox9a [23]. Given the sexually dimorphic pattern of Posox9a and Posox $9 b$ expression, it was possible that Posox $9 a$ and Posox $9 b$ influenced gonadal development by binding to target genes related to sex determination. This study also found the expression of Posox $9 a$ in somatic tissues including gill, muscle and kidney and Posox $9 b$ in the brain and gill, providing further evidence for the idea that the two genes might have an impact on a broad range of biological processes outside the germ line.

\subsection{Posox9a and Posox9b Transcripts Are Detected in Sertoli Cells, Spermatocytes and Oocytes}

The spatial expression profiles in adult Japanese flounder gonads detected by ISH showed that both Posox9a and Posox9b expressed in spermatocytes and Sertoli cells in the testis, and oocytes in the ovary. Sertoli cells are primarily under the hormonal regulation of follicle stimulating hormone (FSH) while fulfilling numerous functions geared toward supporting the development and maturation of germinal cells [24]. The importance of Sertoli cells (and FSH) in sperm production has been emphasized by the demonstration that these cells produce a specific protein, the androgen binding protein (ABP), under FSH control [25]. The expression of Posox9a and Posox $9 b$ in Sertoli cells indicated that they might play roles in sperm production. In the present study, the ISH analysis of ovaries showed that Posox $9 a$ and Posox $9 \mathrm{~b}$ transcripts were detected in the cytoplasm of oocytes, indicating that they might also play a role in the process of oogenesis. 


\subsection{Sexually Dimorphic Expression of Posox9a and Posox9b Mrnas in Gonads during Sex Differentiation}

It has been found via histological observation that the ovarian and testicular differentiation in P. olivaceus juveniles initiated when total length (TL) reached 30 and $37 \mathrm{~mm}[26,27]$, thus juveniles of $60 \mathrm{dah}(32.7 \mathrm{~mm})$ were selected as the initiation of gonadal differentiation in this study [20]. At this critical point of 60 dah, Posox $9 a$ and Posox $9 a$ increased sharply compared with 30 dah, and from then on they both showed obvious sexual dimorphic expression, denoting their important roles in Japanese flounder male sex differentiation. The same expression profiles of Posox9a and Posox9b in the stage of sex differentiation suggested a possible functional redundancy in these domains, which might facilitate gene function ablation for further evaluation of the roles of sox 9 genes in development and evolution. Another possibility is that there might be a direct interaction between the two sox 9 genes and they work together to promote male sex differentiation, which needs further research for verification.

\subsection{7 $\alpha$-methyltestosterone Increased the Expression of Posox9b but Not Posox9a}

The sex of Japanese flounder is easily altered by sex steroid hormone treatment during the period of sex determination [28]. 17 $\alpha$-methyltestosterone, produced by Leydig cells and involved in Wolffian duct differentiation [29], is a familiar steroid hormone for inducing the sex-reverse of genetic females to phenotypic males [27,30]. 17 $\alpha$-methyltestosterone treatment during the period from 30-100 dah, which is the critical period of sex determination and differentiation in Japanese flounder [27], causes the masculinization—gonads differentiated to testes. In zebrafish, at a morphological level, $17 \alpha$-methyltestosterone masculinizes gonads and accelerates spermatogenesis, and these changes are paralleled in the masculinization and de-feminization of gonadal transcriptome. $17 \alpha$-methyltestosterone treatment can upregulate the expression of the genes involved in male sex determination and differentiation [31]. Thus, in order to explore the roles of Posox 9 genes in gonadal function maintenance, we attempt to find whether $17 \alpha$-methyltestosterone administration can influence the expression level of the male-related Posox 9 genes of adult Japanese flounder. In our study, five days after $17 \alpha$-methyltestosterone injection intraperitoneally in 1.5-year-old adults, the expression level of Posox $9 b$ increased significantly compared with the control group, especially when the $17 \alpha$-methyltestosterone concentration was $1.5 \mathrm{mg} / \mathrm{mL}$, whereas the change in Posox $9 a$ was undetectable, manifesting that the effect of androgen on gonadal function maintenance in Japanese flounder might have some relationship with Posox9b but not Posox9a. Nevertheless, further investigations are needed to determine whether $17 \alpha$-methyltestosterone directly regulates the expression of the Posox $9 \mathrm{~b}$ gene. In addition, the different expression profiles of Posox9a and Posox $9 b$ showed that their functions in gonadal development have already diverged to some degree and the detailed differences need to be further explored.

\section{Materials and Methods}

\subsection{Ethics Statement}

Animal experiments were all conducted in accordance with the Regulation for the Administration of Affairs Concerning Experimental Animals (China, 1988). The research was also approved by College of Marine Life, Ocean University of China (Qingdao, China).

\subsection{Fish and Sampling}

All larvae and fish were collected from a commercial hatchery in Haiyang, Shandong Province, China. Larvae, cultivated at $18-21^{\circ} \mathrm{C}$, were sampled at 30, 60, 80 and 100 dah. Ten larvae of each stage were sacrificed for RNA extraction and another ten for histological examination and ISH. The average TL was shown in Table 1 . The whole abdomen that contained the gonadal anlagen was sampled from juveniles with $\mathrm{TL}<50 \mathrm{~mm}$, and gonads were sampled from juveniles with $\mathrm{TL}>50 \mathrm{~mm}$. 
Table 1. The average total length (TL) of sampled P. olivaceus larvae.

\begin{tabular}{ccccccccc}
\hline Period & 30 Dah & 40 Dah & 50 Dah & 60 Dah & 70 Dah & 80 Dah & 90 Dah & 100 Dah \\
\hline TL $(\mathrm{mm})$ & 11.4 & 16.6 & 24.1 & 32.7 & 43.6 & 50.4 & 54.9 & 61.4 \\
\hline
\end{tabular}

Six 1.5-year-old adults (three females and three males) were selected randomly. The body length (234-274 $\mathrm{mm}$ ) and body weight (210-380 g) of those adult individuals were measured. Japanese flounder individuals, anesthetized and sacrificed by severing the spinal cord, were dissected, and the organs, including the heart, liver, spleen, kidney, brain, gill, muscle, intestine and gonad (ovary and testis), were immediately frozen in liquid nitrogen and then stored at $-80^{\circ} \mathrm{C}$ until RNA extraction. Besides, the remaining gonadal tissues were collected and stored in methanol for ISH. Genders were identified through morphological observation.

The 1.5-year-old male Japanese flounder individuals $(n=9)$, injected with androgen ( $17 \alpha$-methyltestosterone) intraperitoneally in concentration of $1.5 \mathrm{mg} / \mathrm{mL}$ and $50 \mu \mathrm{g} / \mathrm{mL}$, were dissected on the fifth day post injection and the testes were immediately frozen in liquid nitrogen and then stored at $-80{ }^{\circ} \mathrm{C}$ until RNA extraction. The control group was injected with an equivalent dosage of ethanol.

\subsection{RNA Extraction and cDNA Synthesis}

RNA were extracted with Trizol Reagent (Invitrogen, Carlsbad, CA, USA) according to the manufacturer's protocol, and then treated with RNase-free DNase I (TaKaRa, Dalian, China) to eliminate genomic DNA contamination. After that, 1.5\% agarose gel electrophoresis and spectrophotometry was used to detect the concentration and integrity. cDNA was synthesized with total RNA and random hexamer primers using a Reverse Transcriptase M-MLV Kit (TaKaRa) following the manufacturer's protocol and verified by PCR using $\beta$-actin gene specific primers (Table 2 ) which spanned different exons.

Table 2. Primers used in this study.

\begin{tabular}{ccc}
\hline Primer Name & Sequence $\mathbf{( 5}^{\prime}-\mathbf{3}^{\prime}$ ) & Usage \\
\hline Posox9a-Fw & ATGAATCTCCTCGACCCTTACC & ORF amplification \\
Posox9a-Rv & TCAGGGTCTGGTGAGCTGG & ORF amplification \\
Posox9a-qRT-Fw & AACGAAGGCGAGAAGCGG & qRT-PCR \\
Posox9a-qRT-Rv & ATGGCATTAGGAGAAATGTGCG & qRT-PCR \\
Posox9a-ISH-Fw & AATCCTCTGTGAGGACTTATTG & ISH probe \\
Posox9a-ISH-Rv & CAGATTCCGTCTCTCTTTCTC & ISH probe \\
Posox9b-Fw & ATCTGTGATACGCTGTTCTTT & ORF amplification \\
Posox9b-Rv & CTCTTCACGGCCTGGAC & ORF amplification \\
Posox9b-qRT-Fw & GCGAGCAAACGCACATA & qRT-PCR \\
Posox9b-qRT-Rv & CCAAAGTCGATGTTGAGCTG & qRT-PCR \\
Posox9b-ISH-Fw & CTTTGAATTGGCTCACAACAG & ISH probe \\
Posox9b-ISH-Rv & ACTGATGACACGGTCATATC & ISH probe \\
amh-qRT-FW & AGCACTGACAGTTTCTCATCC & qRT-PCR \\
amh-qRT-RV & GTAAGACTGATCCCGATGAACTG & qRT-PCR \\
18s-qRT-FW & GGTAACGGGGAATCAGGGT & qRT-PCR \\
18s-qRT-RV & TGCCTTCCTTGGATGTGGT & qRT-PCR \\
$\beta$-actin-qRT-Fw & GAGATGAAGCCCAGAGCAAGAG & qRT-PCR \\
$\beta$-actin-qRT-Rv & CAGCTGTGGTGGTGAAGGAGTAG & qRT-PCR \\
\hline
\end{tabular}

\subsection{Molecular Cloning of Posox9a and Posox $9 b$}

Through searching the de novo transcriptome sequencing data of Japanese flounder [32], we found two different sequences of Japanese flounder sox 9 named Posox $9 a$ and Posox $9 \mathrm{~b}$, respectively. Then, two pairs of primers (Posox9a-Fw/Rv; Posox $9 b-\mathrm{Fw} / \mathrm{Rv}$, Table 2) were designed to confirm the core fragments and ensure sequence accuracy. The total RNA used for obtaining Posox9a and Posox $9 b$ cDNA 
was extracted from adult testis tissue. First-strand cDNA was synthesized from $1 \mu \mathrm{g}$ of total RNA using M-MLV reverse transcriptase (RNase $\left.\mathrm{H}^{-}{ }^{-}\right)(\mathrm{TaKaRa})$ and random primers. The amplified PCR products of the appropriate size were separated by agarose gel electrophoresis, purified using the Zymoclean Gel DNA Recovery kit (Zymo Research, Orange, CA, USA), and cloned into a pMD-19T Vector (TaKaRa) for sequencing.

\subsection{Sequence Analysis}

Sequence data were assembled and analyzed using software suite Lasergene v7.0 (DNASTAR, Madison, WI, USA). Homologous nucleotide and protein sequences were confirmed through using the BLASTn and BLASTx search algorithm in NCBI (http://www.ncbi.nlm.gov/blast). Multiple alignments of amino acid sequences were performed using MUSCLE and refined by GBLOCKS. A phylogenetic tree was generated using maximum likelihood algorithm by MEGA6.0 with a WAG model based on the amino acid sequences analyzed by MUSCLE.

\subsection{Quantitative Real-Time PCR ( $q R T-P C R)$}

The cDNA templates used for qRT-PCR analysis were generated using the method described above and further amplified with $\beta$-actin primers to exclude any possible residual DNA contamination. Primers of Posox9a and Posox9b for qRT-PCR were designed outside the conserved domains to prevent any non-specific amplification. qRT-PCR was performed in a $20 \mu \mathrm{L}$ system containing $10 \mathrm{ng}$ template cDNA, SYBR qPCR SuperMix (Novoprotein, Shanghai, China), $2.5 \mu \mathrm{mol} / \mathrm{L}$ each of specific forward and reverse primers and RNA-free water. Posox $9 a$, Posox $9 b$ and $a m h$ amplicons were separated through $1.5 \%$ agarose gel electrophoresis, purified using the Zymoclean Gel DNA Recovery Kit, cloned into the pMD-19T Vector. The positive clone was verified by sequencing and used for plasmid construction. A standard curve and amplification efficiency were derived from the serial dilutions of the relative plasmid. Melting curves were generated at the end of each run to assess the specificity of the amplicons and the absence of dimers. A negative control (no template) was always included. The result of absolute quantification for the target genes were calculated by the standard curve method with 18S RNA as the reference gene. All qRT-PCR assays for a particular gene were conducted under identical conditions in triplicate. All primers were listed in Table 2.

\subsection{ISH}

ISH of Posox $9 a$ and Posox $9 b$ expression in the gonads was performed using a 393-bp and 459-bp probe spanning the ORF of cDNA, which was amplified by specific primers (Table 2). DIG-labeled RNA sense and anti-sense probes were synthesized using the DIG RNA Labeling Kit (SP6/T7) (Roche, Mannheim, Germany) according to the manufacturer's instructions. ISH on paraffin sections of the gonads was performed according to a previous study [33].

\subsection{Statistical Analysis}

Results were expressed as mean \pm standard error of the mean. qRT-PCR data were analyzed using one-way analysis of variance followed by least significant difference test using SPSS 20.0 (IBM, New York, NY, USA). Significance was set at $p$ value $<0.05$.

\section{Conclusions}

In this study, we found two duplicates of sox 9 in Japanese flounder (Paralichthys olivaceus) named Posox $9 a$ and Posox 9b, respectively. The two genes were conserved in terms of phylogenetic and gene structure analyses. In adult gonads, the expression levels of Posox $9 a$ and Posox $9 b$ were both higher in the testis than the ovary, and their mRNA were both detected in the cytoplasm of oocytes, Sertoli cells and spermatocytes. During sex differentiation, the expression level of Posox $9 a$ and Posox $9 b$ were both higher in male-preferred individuals than female-preferred individuals. $17 \alpha$-methyltestosterone 
injection upregulated the expression of Posox $9 b$, but had no influence on Posox $9 a$ in testis. These results suggested that Japanese flounder Posox $9 a$ and Posox $9 b$ might perform an essential function in gonadal development. Our study provides a foundation for further exploration of the roles of sox 9 genes during the sex determination and differentiation, spermatogenesis and gonadal function maintenance of Japanese flounder.

Acknowledgments: This work was supported by the National Natural Science Foundation of China (No. 31672646), Fundamental Research Funds for the Central Universities (201762016).

Author Contributions: Xiaojing Li and Xubo Wang designed the experiments. Xiaojing Li, Haiyang Yu and Jiangbo Qu performed the experiments. Xiaojing Li, Yujue Wang, Xiaobing Liu and Yuezhong Liu analyzed the data. Xiaojing Li and Xubo Wang wrote the paper. All authors have read and approved the final manuscript.

Conflicts of Interest: The authors declare no conflict of interest.

\section{References}

1. Gubbay, J.; Collignon, J.; Koopman, P.; Capel, B.; Economou, A.; Münsterberg, A.; Vivian, N.; Goodfellow, P.; Lovell-Badge, R.; Gubbay, J.; et al. A gene mapping to the sex-determining region of the mouse $\mathrm{Y}$ chromosome is a member of a new family of embryonically expressed genes. Nature 1990, 346, 245-250. [CrossRef] [PubMed]

2. Sinclair, A.H.; Berta, P.; Palmer, M.S.; Hawkins, J.R.; Griffiths, B.L.; Smith, M.J.; Foster, J.W.; Frischauf, A.M.; Lovellbadge, R.; Goodfellow, P.N. A gene from the human sex-determining region encodes a protein with homology to a conserved DNA-binding motif. Nature 1990, 346, 240-244. [CrossRef] [PubMed]

3. She, Z.Y.; Yang, W.X. Sox family transcription factors involved in diverse cellular events during development. Eur. J. Cell Biol. 2015, 94, 547-563. [CrossRef] [PubMed]

4. Yi, W. The relationship between Sox9 gene and sex determination. Chem. Life Chin. 2000, 20, 70-71.

5. Sarkar, A.; Hochedlinger, K. The sox family of transcription factors: Versatile regulators of stem and progenitor cell fate. Cell Stem Cell 2013, 12, 15-30. [CrossRef] [PubMed]

6. Healy, C.; Uwanogho, D.; Sharpe, P.T. Regulation and role of Sox9 in cartilage formation. Dev. Dyn. 1999, 215, 69-78. [CrossRef]

7. Meyer, J. Autosomal sex reversal and campomelic dysplasia are caused by mutations in and around the sry-related gene Sox9. Cell 1994, 79, 1111-1120.

8. She, Z.Y.; Yang, W.X.; Yang, W.X. Sry and SoxE genes: How they participate in mammalian sex determination and gonadal development? Semin. Cell Dev. Biol. 2016, 63, 13-22. [CrossRef] [PubMed]

9. Kent, J.; Wheatley, S.C.; Andrews, J.E.; Sinclair, A.H.; Koopman, P. A male-specific role for Sox9 in vertebrate sex determination. Development 1996, 122, 2813-2822. [PubMed]

10. Morais, d.S.S.; Hacker, A.; Harley, V.; Goodfellow, P.; Swain, A.; Lovellbadge, R. Sox9 expression during gonadal development implies a conserved role for the gene in testis differentiation in mammals and birds. Nat. Genet. 1996, 14, $62-68$.

11. Moreno-Mendoza, N.; Harley, V.R.; Merchant-Larios, H. Differential expression of Sox9 in gonads of the sea turtle lepidochelys olivacea at male-or female-promoting temperatures. J. Exp. Zool. 1999, 284, 705-710. [CrossRef]

12. Chiang, E.F.; Pai, C.I.; Wyatt, M.; Yan, Y.L.; Postlethwait, J.; Chung, B. Two Sox9 genes on duplicated zebrafish chromosomes: Expression of similar transcription activators in distinct sites. Dev. Biol. 2001, 231, 149-163. [CrossRef] [PubMed]

13. Shen, X.; Cui, J.; Yang, G.; Gong, Q.; Gu, Q. Expression detection of dmrts and two Sox 9 genes in takifugu rubripes (tetraodontidae, vertebrata). J. Ocean Univ. China 2007, 6, 182-186. [CrossRef]

14. Smith, E.K.; Guzmán, J.M.; Luckenbach, J.A. Molecular cloning, characterization, and sexually dimorphic expression of five major sex differentiation-related genes in a scorpaeniform fish, sablefish (Anoplopoma fimbria). Comp. Biochem. Physiol. Part B 2013, 165, 125-137. [CrossRef] [PubMed]

15. Spotila, L.D.; Spotila, J.R.; Hall, S.E. Sequence and expression analysis of wt1 and Sox9 in the red-eared slider turtle, Trachemys scripta. J. Exp. Zool. 1998, 281, 417-427. [CrossRef] 
16. Stolt, C.C.; Lommes, P.; Sock, E.; Chaboissier, M.C.; Schedl, A.; Wegner, M. The Sox9 transcription factor determines glial fate choice in the developing spinal cord. Genes Dev. 2003, 17, 1677-1689. [CrossRef] [PubMed]

17. Spokony, R.F.; Aoki, Y.; Saint-Germain, N.; Magner-Fink, E.; Saint-Jeannet, J.-P. The transcription factor sox9 is required for cranial neural crest development in xenopus. Development 2002, 129, 421-432. [PubMed]

18. Yamaguchi, T.; Yoshinaga, N.; Yazawa, T.; Gen, K.; Kitano, T. Cortisol is involved in temperature-dependent sex determination in the japanese flounder. Endocrinology 2010, 151, 3900-3908. [CrossRef] [PubMed]

19. Yoshinaga, N.; Shiraishi, E.; Yamamoto, T.; Iguchi, T.; Abe, S.-I.; Kitano, T. Sexually dimorphic expression of a teleost homologue of müllerian inhibiting substance during gonadal sex differentiation in japanese flounder, Paralichthys olivaceus. Biochem. Biophys. Res. Commun. 2004, 322, 508-513. [CrossRef] [PubMed]

20. Liu, Y.; Zhang, W.; Du, X.; Zhao, J.; Liu, X.; Li, X.; Zhang, Q.; Wang, X. Sexually dimorphic expression in developing and adult gonads shows an important role of gonadal soma-derived factor during sex differentiation in olive flounder (Paralichthys olivaceus). Comp. Biochem. Physiol. Part B 2017, 210, 1-8. [CrossRef] [PubMed]

21. Barrionuevo, F.; Georg, I.; Scherthan, H.; Lécureuil, C.; Guillou, F.; Wegner, M.; Scherer, G. Testis cord differentiation after the sex determination stage is independent of Sox9 but fails in the combined absence of Sox9 and Sox8. Dev. Biol. 2009, 327, 301-312. [CrossRef] [PubMed]

22. Dong, X.; Chen, S.; Ji, X.; Shao, C. Molecular cloning, characterization and expression analysis of Sox $9 a$ and Foxl2 genes in half-smooth tongue sole (Cynoglossus semilaevis). Acta Oceanol. Sin. 2011, 30, 68-77. [CrossRef]

23. Raghuveer, K.; Senthilkumaran, B. Isolation of Sox 9 duplicates in catfish: Localization, differential expression pattern during gonadal development and recrudescence, and hcg-induced up-regulation of Sox9 in testicular slices. Reproduction 2010, 140, 477-487. [CrossRef] [PubMed]

24. Gorczynska, E.; Handelsman, D. The role of calcium in follicle-stimulating hormone signal transduction in Sertoli cells. J. Biol. Chem. 1991, 266, 23739-23744. [PubMed]

25. French, F.; Ritzen, E. Androgen-binding protein in efferent duct fluid of rat testis. J. Reprod. Fertil. 1973, 32, 479-483. [CrossRef] [PubMed]

26. Tanaka, H. Gonadal sex differentiation in flounder, Paralichthys olivaceus. Bull. Natl. Res. Inst. Aquac. 1987, 11, 7-19.

27. Yamamoto, E. Studies on sex-manipulation and production of cloned populations in hirame, Paralichthys olivaceus (temminck et schlegel). Aquaculture 1999, 173, 235-246. [CrossRef]

28. Kitano, T.; Takamune, K.; Nagahama, Y.; Abe, S.-I. Aromatase inhibitor and 17a-methyltestosterone cause sex-reversal from genetical females to phenotypic males and suppression of $\mathrm{p} 450$ aromatase gene expression in japanese flounder (Paralichthys olivaceus). Mol. Reprod. Dev. 2000, 56, 1-5. [CrossRef]

29. Josso, N.; Cate, R.; Picard, J.; Vigier, B.; di Clemente, N.; Wilson, C.; Imbeaud, S.; Pepinsky, R.; Guerrier, D.; Boussin, L. Anti-müllerian hormone: The jost factor. Recent Prog. Horm. Res. 1993, 48, 1-59. [PubMed]

30. Hoar, W.S.; Randall, D.J.; Donaldson, E.M. Fish Physiology; Academic Press: Cambridge, MA, USA, 1983.

31. Fisher, A.; Horsfield, J.A.; Rutherford, K.; Black, M.A.; Gemmell, N.J.; Lee, S.L.J. Histological and transcriptomic effects of $17 \alpha$-methyltestosterone on zebrafish gonad development. BMC Genom. 2017, 18, 557.

32. Wang, W.; Wang, J.; You, F.; Ma, L.; Yang, X.; Gao, J.; He, Y.; Qi, J.; Yu, H.; Wang, Z. Detection of alternative splice and gene duplication by rna sequencing in japanese flounder, Paralichthys olivaceus. G3 2014, 4, 2419-2424. [CrossRef] [PubMed]

33. Gao, J.; Wang, J.; Jiang, J.; Fan, L.; Wang, W.; Liu, J.; Zhang, Q.; Wang, X. Identification and characterization of a nanog homolog in japanese flounder (Paralichthys olivaceus). Gene 2013, 531, 411-421. [CrossRef] [PubMed]

(C) 2018 by the authors. Licensee MDPI, Basel, Switzerland. This article is an open access article distributed under the terms and conditions of the Creative Commons Attribution (CC BY) license (http:// creativecommons.org/licenses/by/4.0/). 